\title{
Characterization of High Yield Graphene Oxide Synthesized by Simplified Hummers Method*
}

\author{
Kayode Oladele Olumurewa, Bolutife Olofinjana, Oladepo Fasakin, Marcus Adebola Eleruja\#, \\ Ezekiel Oladele Bolarinwa Ajayi
}

Department of Physics and Engineering Physics, Obafemi Awolowo University, Ile-Ife, Nigeria

Email: "meleruja@oauife.edu.ng

How to cite this paper: Olumurewa, K.O., Olofinjana, B., Fasakin, O., Eleruja, M.A. and Ajayi, E.O.B. (2017) Characterization of High Yield Graphene Oxide Synthesized by Simplified Hummers Method. Graphene, 6, 85-98.

https://doi.org/10.4236/graphene.2017.64007

Received: August 16, 2017

Accepted: October 28, 2017

Published: October 31, 2017

Copyright $\odot 2017$ by authors and Scientific Research Publishing Inc. This work is licensed under the Creative Commons Attribution International License (CC BY 4.0).

http://creativecommons.org/licenses/by/4.0/

(c) (i) Open Access

\begin{abstract}
Graphene oxide (GO) was chemically synthesized from natural flake graphite (NFG) using the simplified Hummers method. The synthesis was carried out using two routes. The first route involved stirring the one pot mixture continuously for three days at ambient temperature while the second route involved stirring another one pot mixture for six days also at ambient temperature. The two GOs were characterized using Fourier Transform Infrared Spectroscopy (FTIR), Energy Dispersive X-Ray Spectroscopy (EDX), Field Emission Scanning Electron Microscopy (FE-SEM), Raman Spectroscopy and UV-Visible Spectrometry. The FTIR spectra showed introduction of oxygen functionalities in both GO with a higher degree of oxidation in the 6-day synthesized GO while the EDX confirmed the presence of carbon and oxygen in the GOs. The SEM micrograph gave the typical wrinkle and crumpling present in the 3-day synthesized GO while the 6-day synthesized GO showed distortion in structures. The Raman spectra showed a slightly higher $\mathrm{I}_{\mathrm{D}} / \mathrm{I}_{\mathrm{G}}$ ratio for the 3-day synthesized GO with the 6-day synthesized GO showing a greater disruption of the $\mathrm{sp}^{2}$ domains. The extended period of stirring and oxidation increased the band gap of the 6-day synthesized GO to $3.0 \mathrm{eV}$ unlike the 3-day synthesized GO where $2.5 \mathrm{eV}$ was observed.
\end{abstract}

\section{Keywords}

Graphite, Graphene, Graphene Oxide, Raman Spectroscopy, Band Gap

\section{Introduction}

Graphene is a versatile two-dimensional material with various applications ranging from electronic devices to biological devices [1]. It is a nanoscale allotrope of carbon rolled into one-dimensional nanotubes or folded into ze*Effect of extended oxidation on the properties of GO. 
ro-dimensional fullerenes [2]. As a single aromatic sheet of $\mathrm{sp}^{2}$ bonded carbon, it exhibits novel electronic properties such as ballistic transport and high conductivity [3]. Graphene can be synthesized by various methods such as chemical vapour deposition, mechanical exfoliation and cleavage and annealing of single-crystal $\mathrm{SiC}$ under ultrahigh vacuum. The high energy requirement and low yield serve as a limitation in utilizing these methods [4]. Chemical oxidation of graphite is the most utilized synthesis method for graphene oxide (GO) [1] and the process introduces functional groups such as carbonyl, hydroxyl and peroxyl in between carbon layers of graphite [5]. Graphene oxide (GO) can be produced using inexpensive graphite as raw material through the chemical method which is cost-effective [6]. The chemical method involves oxidation of graphite to GO using highly oxidizing reagents. Formation of large quantity of GO in powder form is an advantage [1] as well as its simplicity and cost effectiveness [4]. The process involves oxidation of graphite and exfoliating into monolayers or few layered stacks of GO [4].

The popular Hummers method usually requires several steps, tedious and long experimental time and temperature control for the preparation of GO, hence the need to find a simpler method of oxidizing GO. In this work, the simplified hummers method was utilized to synthesize GO whereby the long hours of mixing have been mitigated to just 5 minutes of mixing and several temperature controls have been reduced to stirring at room temperature continuously. We also decided to investigate the characteristics of the GO obtained when the mixture is stirred continuously for three days and for six days. Fourier Transform Infrared Spectroscopy (FTIR), Energy Dispersive X-Ray Spectroscopy (EDX), Field Emission Scanning Electron Microscopy (FE-SEM), Raman Spectroscopy and UV-Visible Spectrometry were utilized to determine these characteristics.

\section{Experimental Method}

\subsection{Synthesis of Graphene Oxide}

Graphene oxide was prepared using the simplified Hummers' method. $3 \mathrm{~g}$ flake graphite (44 $\mu \mathrm{m}, 99.8 \%$ carbon, Ash colour from Asbury Carbon, New Jersey, USA) was added into $400 \mathrm{~mL}$ solution of concentrated $\mathrm{H}_{2} \mathrm{SO}_{4}(98 \%, \mathrm{BDH})$ in $1000 \mathrm{~mL}$ conical flask. The mixture of the flake graphite and acid was placed in an ice-bath in order to keep the temperature below $10^{\circ} \mathrm{C}$ and put under vigorous magnetic stirring for five minutes at a constant rate while $18 \mathrm{~g}$ of $\mathrm{KMnO}_{4}$ was gradually added into the solution with continuous stirring (Figure 1). After adding the $\mathrm{KMnO}_{4}$, the solution turned purplish green which typifies the reaction of $\mathrm{KMnO}_{4}$ with the acidic solution. The reaction continued for three days while stirring continuously at ambient temperature. After three days, the solution turned deep green. The procedure was also repeated with stirring continuously for six days in another separate set up. After six days, the green colour of the mixture was more intense. For each of the GO, the mixture was poured into a $2 \mathrm{~L}$ conical flask containing $400 \mathrm{~mL}$ of cold deionized water. While pouring 


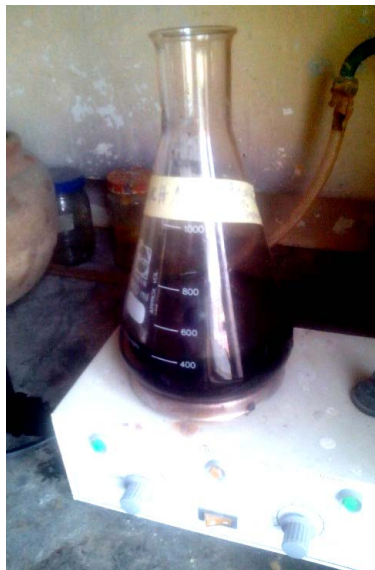

(a)

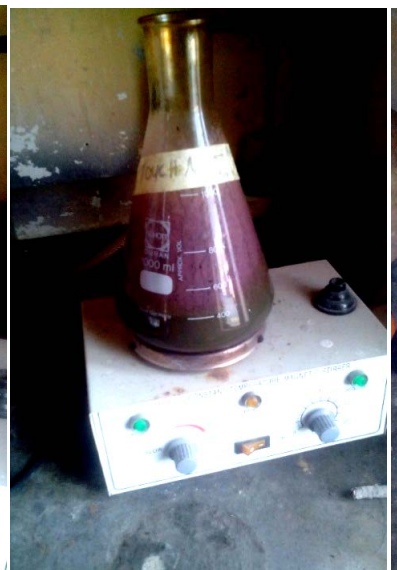

(b)

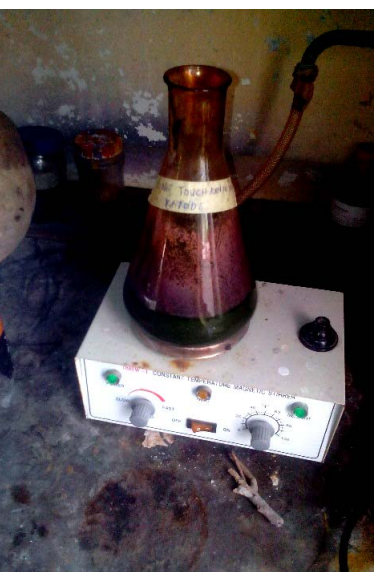

(c)

Figure 1. (a) Mixture of graphite flakes, $\mathrm{H}_{2} \mathrm{SO}_{4}$ and $\mathrm{KMnO}_{4}$ at the onset of stirring; (b) Resulting mixture after three days of stirring; (c) Resulting mixture after six days of stirring.

the mixture, an exothermic reaction ensued with effervescence occurring and the mixture turned reddish brown. In order to stop the reaction, $27 \mathrm{~mL}$ of $\mathrm{H}_{2} \mathrm{O}_{2}$ $(30 \% \mathrm{BDH})$ was added to the mixture and the colour changed from reddish brown to light yellow. The mixture was then stirred for ten minutes and the colour changed to dark brown. This shows that oxidized graphite has been synthesized. The mixture was then centrifuged and washed with $1 \mathrm{M} \mathrm{HCl}$ three times and washed several times with deionized water till a $\mathrm{pH}$ of between $4-5$ was obtained. During the washing process with deionized water, the graphite oxide experienced exfoliation, which resulted in the thickening of the graphene solution, forming a GO gel. The mixture was filtered while the oven drying method and the freeze drying method were both employed in order to compare the two drying routes. Subsequently, the freeze dried and oven dried GO were pulverized. The powder obtained was sieved to obtain even particle size. Hence, we have two different graphene oxide, differing in the number of days allowed for stirring and oxidation. We have the 3-day synthesized GO and the 6-day synthesized GO. Both samples were characterized to investigate the effect of extended stirring and oxidation on the material.

\subsection{Characterization of the Synthesized GOs}

Infrared spectrum of GO was obtained using Fourier Transform Infrared Spectrophotometer (FTIR, SHIMADZU IRTracer-100). The compositional ratio of the synthesized GO was determined using Energy Dispersive X-ray (EDX) set up. The surface morphology characterization was carried out using Zeiss Ultra Plus 55 Field Emission Scanning Electron Microscope (FE-SEM) while the Optical absorbance of the liquid GO was carried out using Jenway UV-Visible Spectrophotometer (Model 6405). The Raman Spectroscopy analysis was carried out using Jobin-Yvon Horiba T64000 micro-Raman Spectrometer equipped with a triple monochromator system to eliminate contributions from Rayleigh scat- 
tering.

\section{Results and Discussion}

\subsection{FTIR Spectroscopy Analysis of GOs}

Figure 2(a) shows the spectrum of natural flake graphite (NFG) and Figure 2(b) typifies the spectrum of graphene oxide synthesized during a three day procedure of stirring continuously while Figure 2(c) indicates graphene oxide synthesized during a procedure of stirring for six days. The spectra however show certain differences. In Figure 2(a) NFG spectrum shows characteristic bands typical of NFG, for example; O-H broad peak at $3433 \mathrm{~cm}^{-1}$ resulting from intercalated water and $\mathrm{C}=\mathrm{C}$ at $1629 \mathrm{~cm}^{-1}$ which can be assigned to the skeletal vibrations of graphitic domains.

In Figure 2(b), the 3-day GO spectrum show bands at $3423 \mathrm{~cm}^{-1}$ for $\mathrm{O}-\mathrm{H}$ stretching, at $3064 \mathrm{~cm}^{-1}$ and $3236 \mathrm{~cm}^{-1}$ the bands are due to $\mathrm{sp}^{2} \mathrm{C}-\mathrm{H}$ stretching frequency, at 2881 and $2804 \mathrm{~cm}^{-1}$ the bands typify $\mathrm{sp}^{3} \mathrm{C}-\mathrm{H}$. The most intense peak occurs between $3320 \mathrm{~cm}^{-1}$ and $3430 \mathrm{~cm}^{-1}$ and is attributed to the $\mathrm{C}-\mathrm{OH}$ stretching vibrations of a hydroxyl group. The bands at $1712 \mathrm{~cm}^{-1}$ region is due to the $\mathrm{C}=\mathrm{O}$ stretching vibrations of a carbonyl group. The spectrum also has bands at $1616 \mathrm{~cm}^{-1}$ due to $\mathrm{C}=\mathrm{C}$ which is a similar peak with graphite, signifying skeletal vibrations of non-exfoliated graphite. The band at $1232 \mathrm{~cm}^{-1}$ typifies presence of epoxides and its asymmetric epoxide bands at $875 \mathrm{~cm}^{-1}$. Between $1040 \mathrm{~cm}^{-1}$ and $1170 \mathrm{~cm}^{-1}$ are peaks that are attributed to the alkoxy C-O stretching vibration. The widest peak is located at $1232 \mathrm{~cm}^{-1}$ and is attributed to the stretching vibration of the epoxy group $\mathrm{C}-\mathrm{O}-\mathrm{C}$ [7]. There is absence of the $\mathrm{C}=\mathrm{C}$ peak which typically occurs at $1570 \mathrm{~cm}^{-1}$. The $\mathrm{C}=\mathrm{C}$ bond missing indicated a strong oxidation have taken place [8]. The appearance of broad peak between $900-1200 \mathrm{~cm}^{-1}$ is attributed to C-O stretching with a C-O-C peak at $1232 \mathrm{~cm}^{-1}$ and $\mathrm{C}-\mathrm{H}$ peak at $1398 \mathrm{~cm}^{-1}$ [9].

In the 6-day synthesized GO as shown in Figure 2(c), the spectrum is similar to that of 3-day synthesized GO except for the absence of a $\operatorname{sp}^{2} \mathrm{CH}$ and a $\mathrm{C}=\mathrm{O}$ bond making it appear like an alcohol and not a carboxyllic group. We can therefore infer that a longer period of oxidation destroys the $\mathrm{sp}^{2}$ network on the GO. The epoxides are also absent in this spectrum unlike in Figure 2(b) where they are strongly present. The more intense $\mathrm{C}-\mathrm{O}$ peak at $1093 \mathrm{~cm}^{-1}$ is suggestive of a higher degree of oxidation in the 6-day synthesized GO compared to that obtained in the 3-day synthesized GO. Cao et al., (2012) reported that fully oxidized stochiometric GO's do not contain a significant fraction of $\mathrm{sp}^{2}$ carbon atoms which are responsible for bandgap opening and related optical properties. The higher disruption of the $\mathrm{sp}^{2}$ network in the 6-day synthesized GO may cause an increase in defect in this synthesized graphene oxide as well as a lower conductivity compared to that obtained in the 3-day synthesized GO. This may be attributed to the extended time of oxidation reaching to higher oxidation [10]. However, the two spectrum typify the presence of $\mathrm{OH}$ group, $\mathrm{C}=\mathrm{C}$ bond, $\mathrm{sp}^{3} \mathrm{CH}$ 


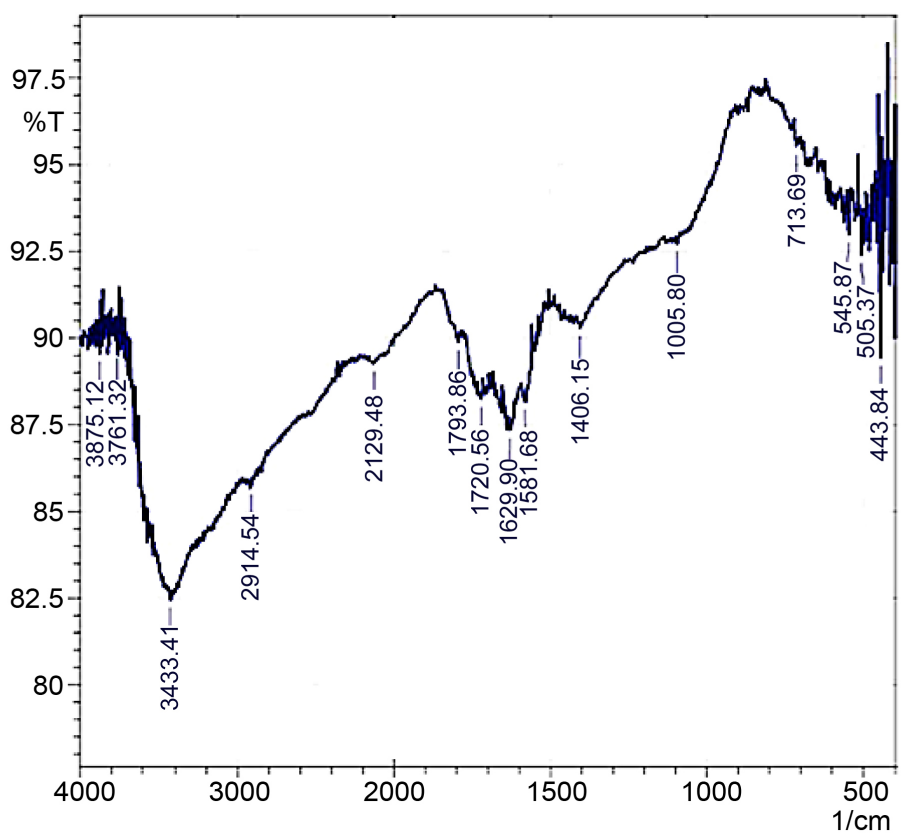

(a)

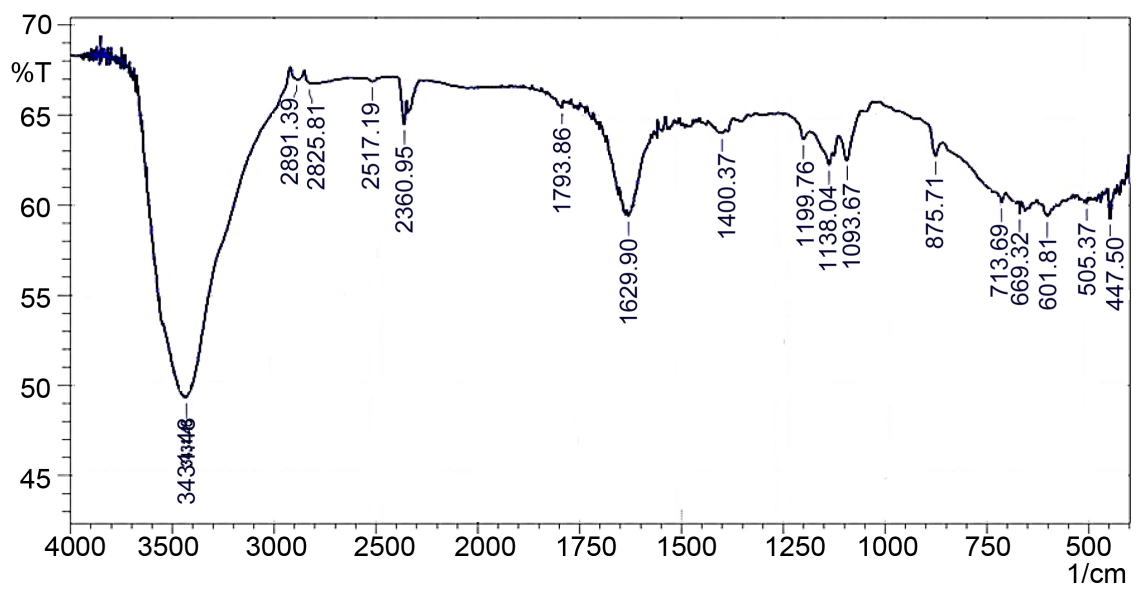

(b)

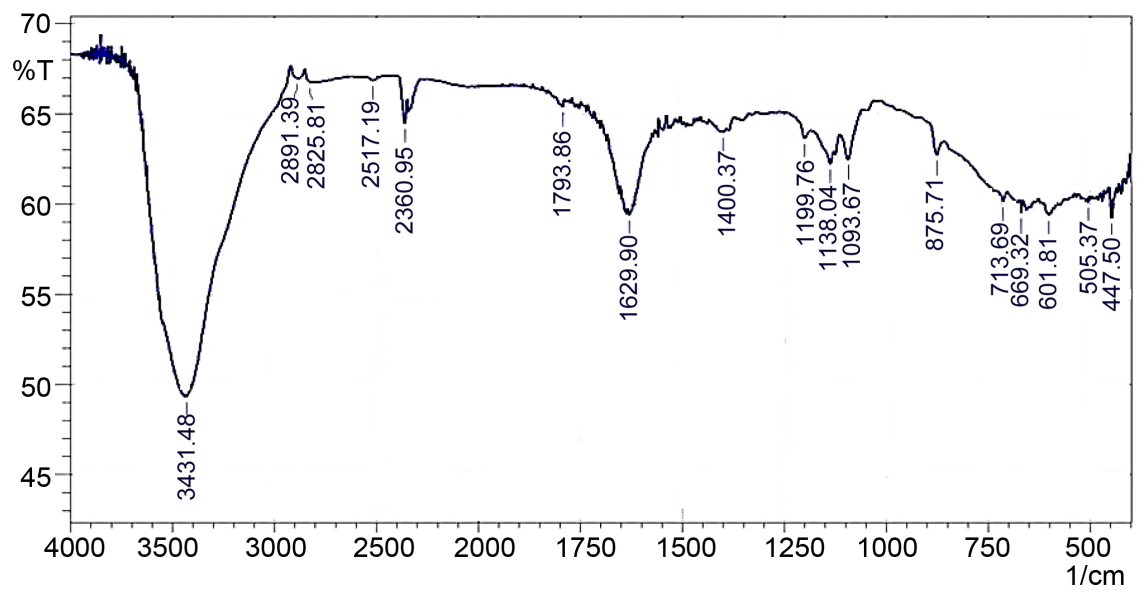

(c)

Figure 2. FTIR spectrum of (a) natural flake graphite powder, (b) 3-day synthesized GO gel, (c) 6-day synthesized GO gel. 
and $\mathrm{C}-\mathrm{O}$ bond.

\subsection{Energy Dispersive X-Ray Analysis of GO}

Figure 3 shows the typical EDX spectrum of the synthesized GOs. The spectrum shows the presence of only carbon and oxygen thereby confirming the synthesis of graphene oxide. The lack of precipitate after centrifugation during the synthesis of the GOs suggests that $100 \%$ or near $100 \%$ conversion of graphite to GO was achieved [1]. This is observable as seen in the high oxygen content of the GO.

\subsection{Scanning Electron Microscope Analysis of GO Powder}

The microstructure and surface analysis of the synthesized GOs were carried out using FE-SEM and are shown in Figure 4 and Figure 5. In Figure 4 the three day synthesized GO shows typical crumpled and wrinkled graphene sheet structure which was the result of deformation upon exfoliation and restacking process whereby the morphology resembles a thin curtain [11]. The six days synthesized GO however showed distortion in micro structure as the typical wrinkle of the graphene layer is not noticeable.

Figure 5 shows the micrograph of freeze dried GO and oven dried GO. The structures in the freeze dried GO are more preserved as shown by the wrinkling and crumpling typical of GO in the freeze dried GO unlike in the oven dried GO where the sheets are stacked upon one another like a multilayer structure. The freeze dried GO shows that the oxygen functional group has prevented it from stacking [12]. A highly roughened surface for the freeze dried GO sample is due to the freeze drying treatment and is in contrast to the smoother surface of the oven dried GO [13].

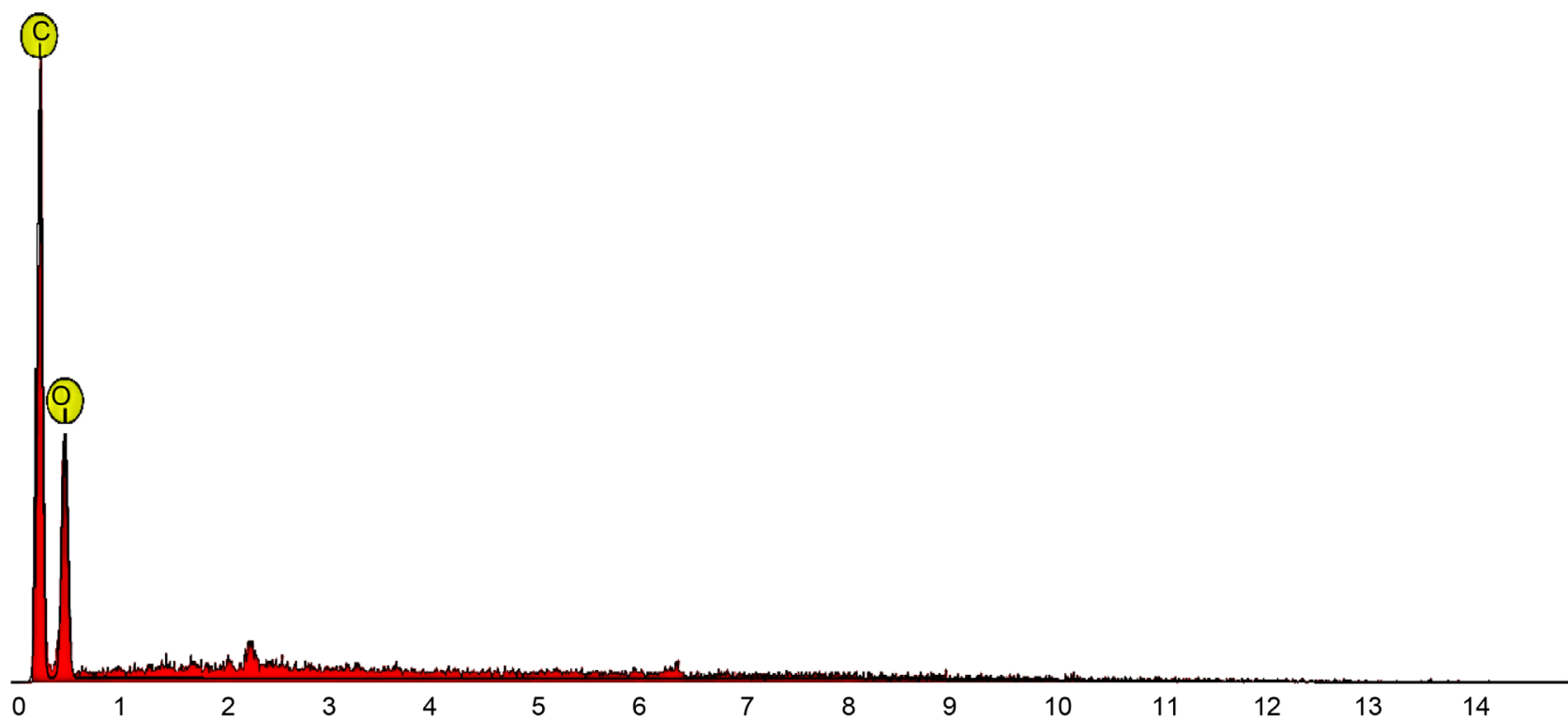
21,620 counts in 30 seconds

Figure 3. Typical EDX spectrum of synthesized GOs. 


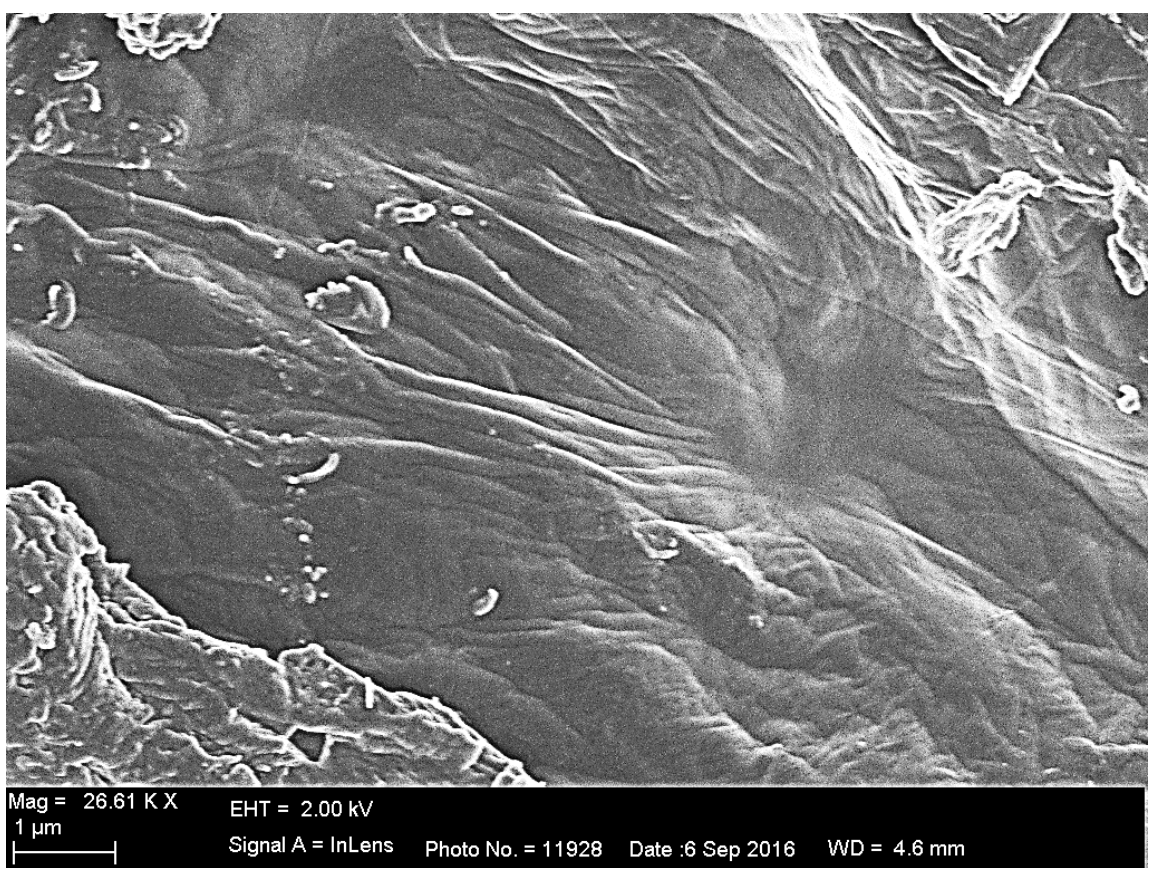

(a)

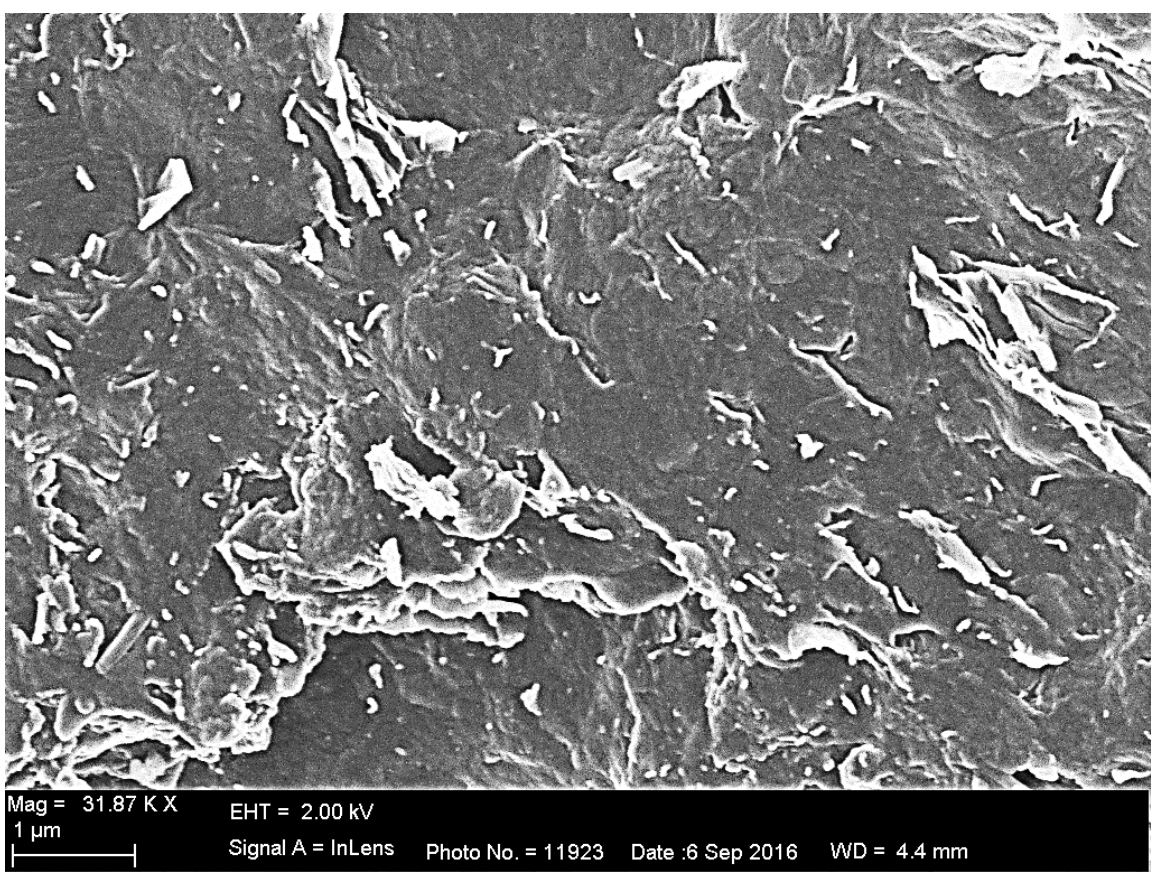

(b)

Figure 4. FE-SEM micrograph of (a) 3-day synthesized GO and (b) 6-day synthesized GO.

\subsection{Raman Spectroscopy Analysis of G0 Powder}

Figure 6 shows Raman spectra of the synthesized GOs. In Raman spectroscopy, $D$ band indicates the extent of defects whereas $G$ band indicates the graphitic nature. If the $\mathrm{D}$ peak intensity is higher, the sample has more defects in graphite network. These defects will be imparted to the edges of the sheets. If the G band is prominent, the sample is said to be crystalline [14]. The D and G peaks are 


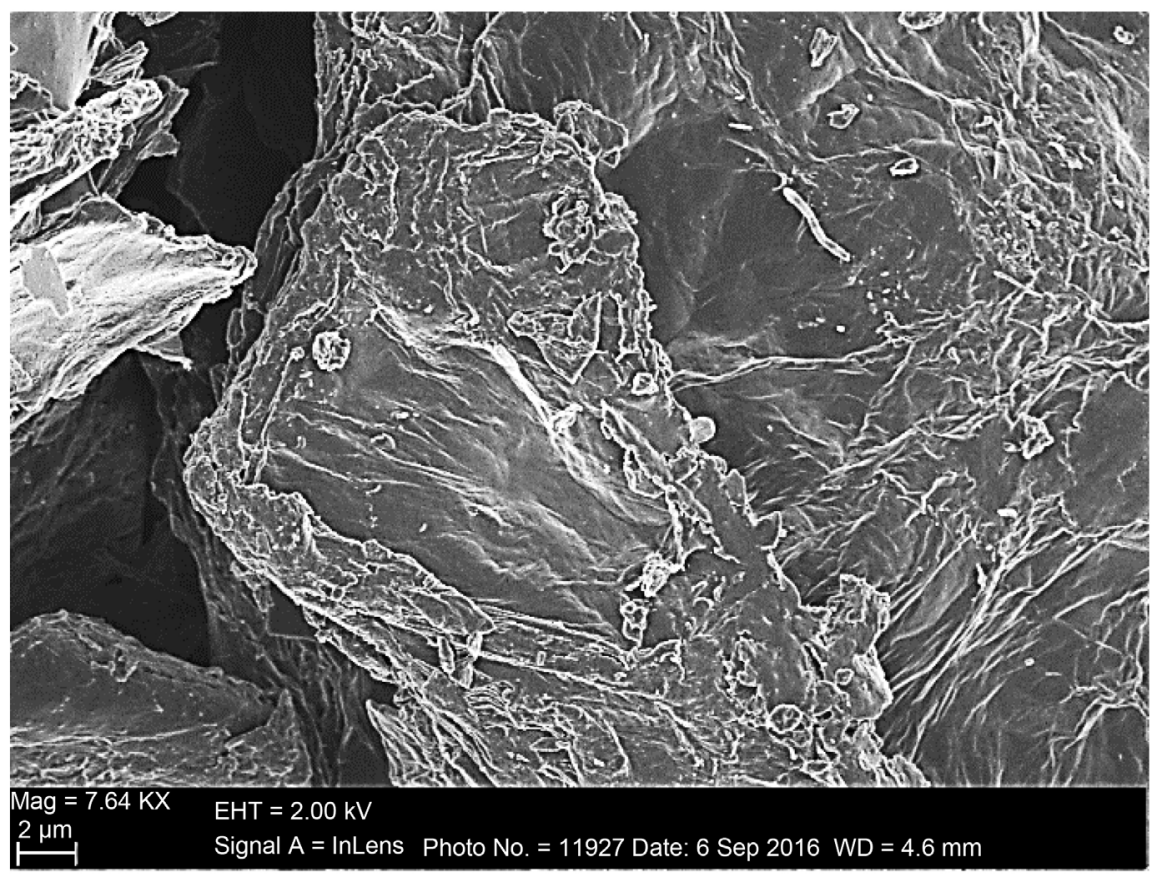

(a)

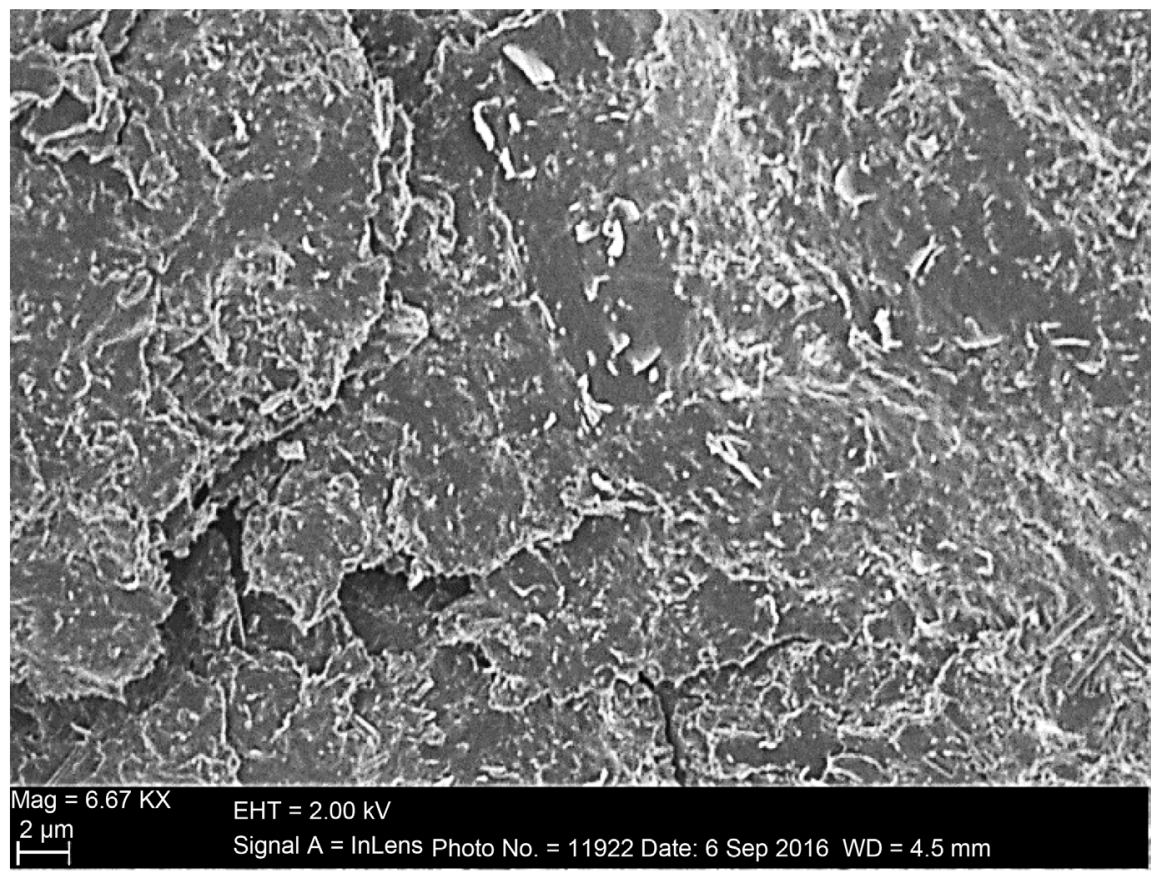

(b)

Figure 5. FE-SEM micrograph of (a) freeze dried GO and (b) oven dried GO.

higher in the 3-day synthesized GO compared to the 6-day synthesized GO. A higher intensity in the D band of the 3-day synthesized GO shows that the material has more defect in the graphitic network compared to the 6-day synthesized GO [14]. The higher intensity of the G band in the 3-day synthesized GO typifies a more crystalline material compared to the 6-day synthesized GO. However, the ratio of ID to IG for both synthesis are quite close at 1.01 for 3-day 


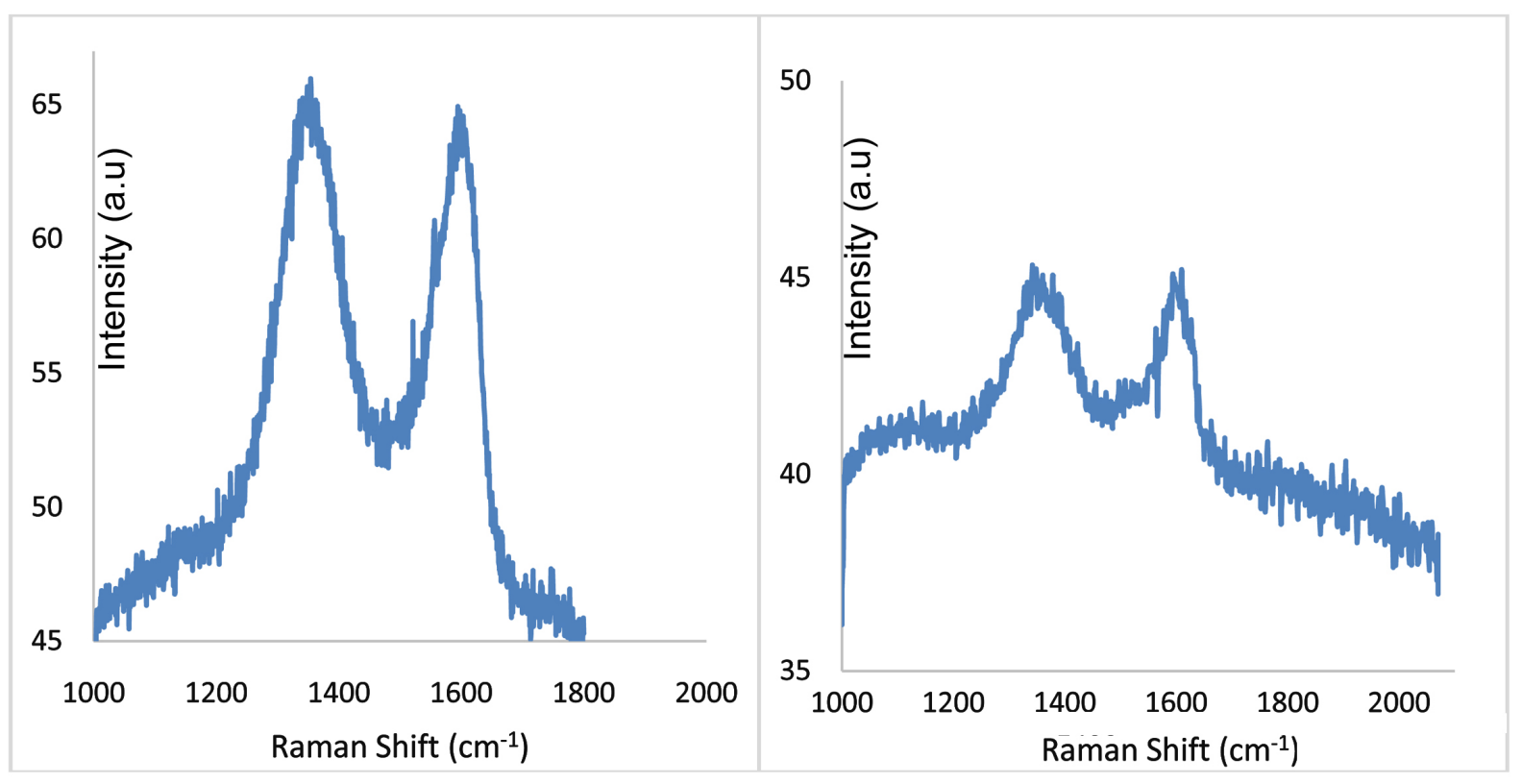

(a)

(b)

Figure 6. Raman spectra of (a) 3-day synthesized GO and (b) 6-day synthesized GO.

synthesized GO and 1.00 for 6-day synthesized GO. The relative intensity ratio of both peaks (ID/IG) in the two synthesis method is a measure of disorder degree and is inversely proportional to the average size of the $\mathrm{sp}^{2}$ cluster [15]. It can thus be inferred that there is equal average size of the $\mathrm{sp}^{2}$ in both samples and by deduction, the extended period of oxidation did not alter the average size of the $\mathrm{sp}^{2}$. The D peak of 3-day synthesized GO located at $1354 \mathrm{~cm}^{-1}$ and that of the 6-day synthesized GO located at $1348 \mathrm{~cm}^{-1}$ emanated from a defect-induced breathing mode of $\mathrm{sp}^{2}$ rings. It is common to all $\mathrm{sp}^{2}$ carbon lattice and arises from the stretching of $\mathrm{C}-\mathrm{C}$ bond. The G-peak of the 3-day synthesized GO and 6-day synthesized GO at around $1603 \mathrm{~cm}^{-1}$ and $1608 \mathrm{~cm}^{-1}$ respectively is due to the first order scattering of the E2g phonon of $\mathrm{sp}^{2}$ carbon domains [16]. The increase of the D peak intensity in the 3-day synthesized GO compared to 6-day synthesized GO indicate presence of more $\mathrm{sp}^{2}$ domains. Hence, the extended period of oxidation brought about a greater disruption of the $\mathrm{sp}^{2}$ domains in the 6-day synthesized GO.

\subsection{Optical Characterization of GO}

Figure 7(a) and Figure 7(b) give the spectrum of absorbance against wavelength for the 3-day synthesized GO and 6-day synthesized GO. The spectra of absorbance against wavelength showed that the 6-day synthesized GO had lower absorbance and by implication higher transmittance when compared to 3-day synthesized GO. At a wavelength of $228 \mathrm{~nm}$, 6-day synthesized GO had an absorbance of 0.666 while at almost the same wavelength of $227 \mathrm{~nm}$, 3-day synthesized GO had an absorbance of 1.203. It has been reported that graphene based colloids can exhibit interesting absorption patterns at the wavelength regions of 


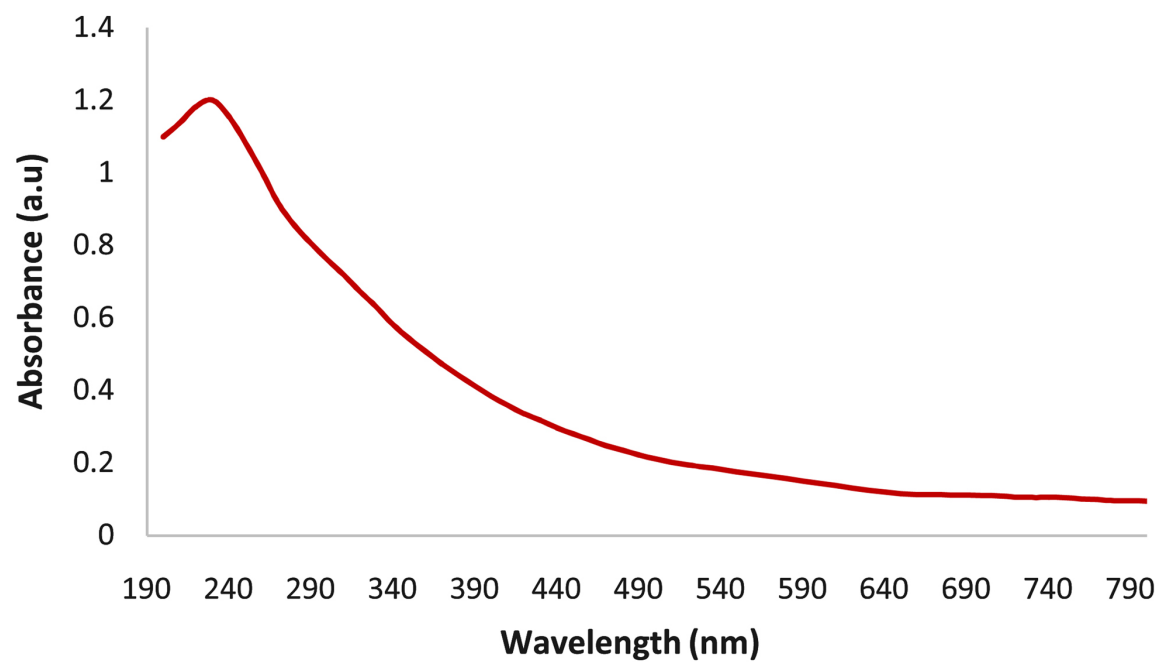

(a)

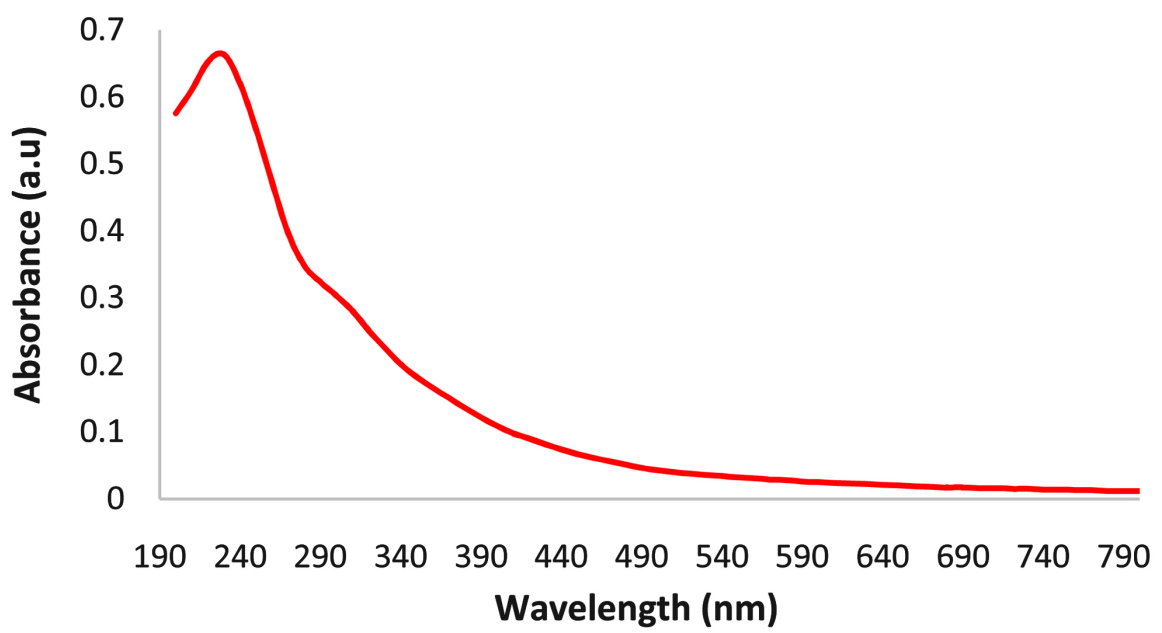

(b)

Figure 7. UV-Visible spectra of (a) 3-day synthesized GO, (b) 6-day synthesized GO.

$\sim 230 \mathrm{~nm}, \sim 260 \mathrm{~nm}$ and $\sim 300 \mathrm{~nm}$ corresponding to transitions involving $\pi, \sigma$ and $\mathrm{n}$ electrons of different bond energies [16]. The two GO samples, produced a characteristic peak at $\sim 230 \mathrm{~nm}$ which is attributed to $\pi-\pi^{*}$ plasmon of the $\mathrm{C}=\mathrm{C}$ bonds. We also observed a shoulder peak at $\sim 300 \mathrm{~nm}$ for both samples though more pronounced in the 6-day synthesized GO which suggests that there exists a bond excitation value at $\sim 300 \mathrm{~nm}$ corresponding to $\mathrm{n}-\pi^{*}$ transition of the $\mathrm{C}=\mathrm{O}$ chromophores for aldehyde and ketone molecule [17].The higher the ratio of the intensity of $\mathrm{C}=\mathrm{C}$ bonding peak occurring at $\sim 230 \mathrm{~nm}$ to the intensity occurring at $300 \mathrm{~nm}$ peak, the higher the degree of oxidation. This is because a more oxidized graphene basal plane yields a greater amount of isolated aromatic rings that increase the intensity of $\mathrm{C}=\mathrm{C}$ bonding peak (1). In our research, the 3 -day synthesized GO has a ratio of 1.58 while the 6-day synthesized GO has a ratio of 2.18. Thus, the 6-day synthesized GO typifies a higher degree of oxidation. The $\mathrm{UV}$ results of intensity ratio of $\mathrm{C}=\mathrm{C}$ peak to ratio of $\mathrm{C}=\mathrm{O}$ peak of $\mathrm{GO}$ reported 
in several literatures gave a ratio less than 2 [16] [17]. The absorption coefficient is given by

$$
\alpha=1 / d \ln 1 / T
$$

where $d$ is the thickness of the film and $T$ is the transmittance which is equal to $10-\mathrm{A}$ and $\mathrm{A}$ is the absorbance. Figure 8 shows the plot of square of absorption coefficient ( 22 ) against the energy of the photon for the 3-day synthesized GO and the 6-day synthesized GO. The extrapolation of the linear part of the graphs gave a bandgap of $2.5 \mathrm{eV}$ and $3.0 \mathrm{eV}$ for the 3-day synthesized GO and the 6-day synthesized GO respectively. The reduction in bandgap of the 3-day synthesized

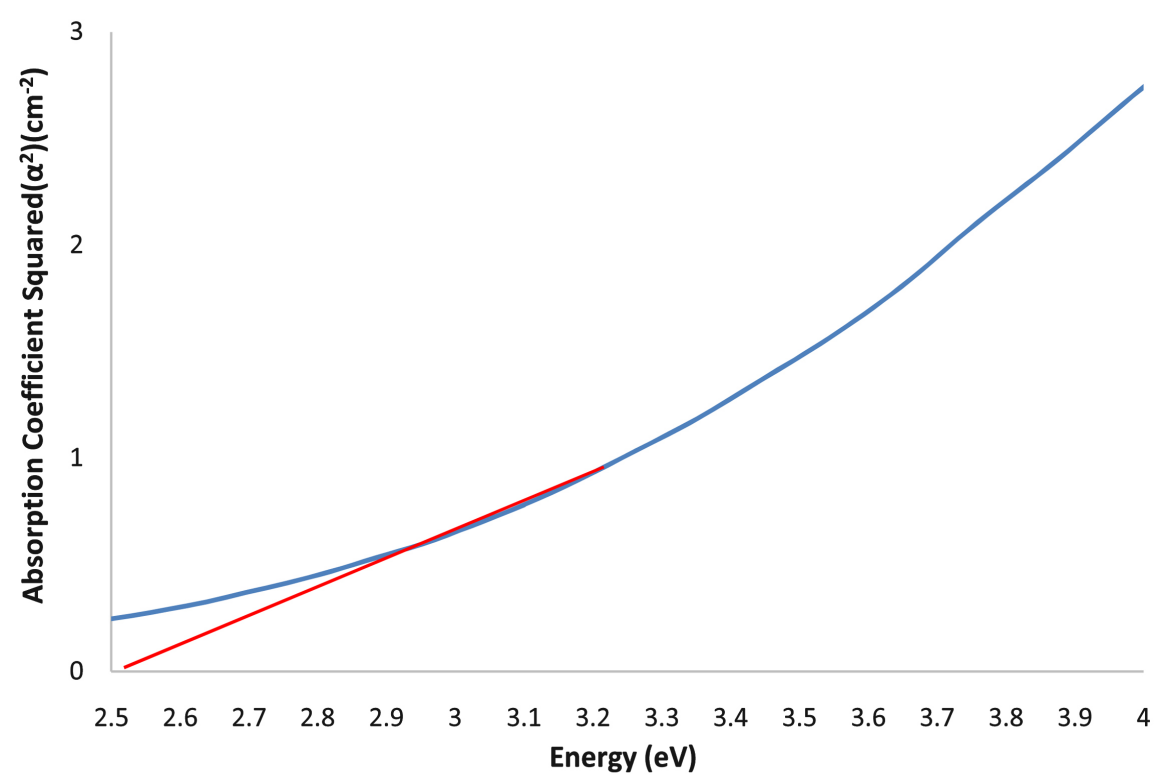

(a)

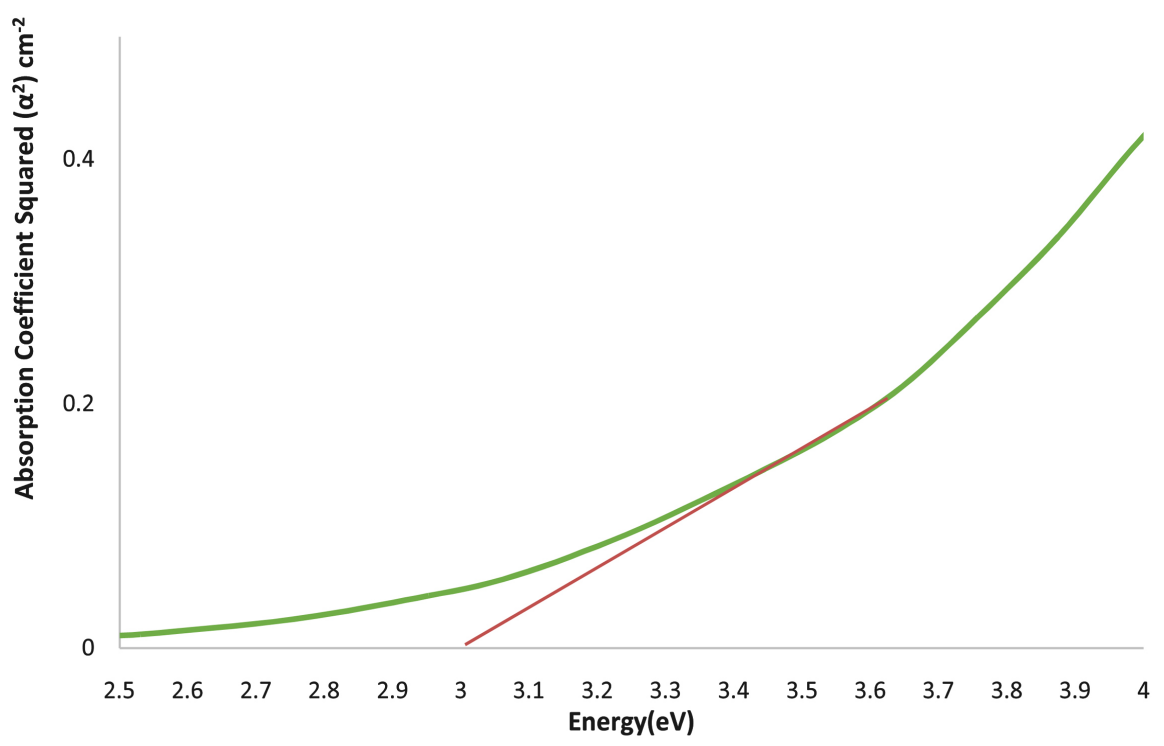

(b)

Figure 8. Square of absorption coefficient $\left(\alpha^{2}\right)$ vs. energy of (a) 3-day synthesized GO, (b) 6-day synthesized GO. 
GO compared to the 6-day synthesized GO can lead to better conductivity. We can infer that the 3-day synthesized GO may be more conducting than the 6-day synthesized GO, hence extended period of oxidation is not good for synthesizing GOs that will be used as electrodes or battery materials.

\section{Conclusions}

Graphene oxide was synthesized using the simplified Hummers method by oxidizing for three days and six days. The two samples of GO were analysed to investigate the functional groups, compositional, morphology, optical and structural properties.

FTIR spectrum showed similar spectrum for the 3-day synthesized GO and the 6-day synthesized GO. However, for the 6-day synthesized GO the absence of a sp ${ }^{2} \mathrm{CH}$ and a $\mathrm{C}=\mathrm{O}$ band confirms that a longer period of oxidation destroys the $\mathrm{sp}^{2}$ network on the GO. The more intense C-O peak in the 6-day synthesized $\mathrm{GO}$ is suggestive of a higher degree of oxidation.

The EDX result confirmed the oxidation of graphite flakes with presence of carbon and oxygen peaks. The SEM micrograph showed that the extended period of oxidation distorted the graphene sheets in the 6-day synthesized GO unlike in the 3-day synthesized GO where we observed the typical crumpling and wrinkling structure.

The Raman spectra showed that more $\mathrm{sp}^{2}$ domains are present in the 3-day synthesized GO compared to 6-day synthesized GO. Hence, the extended period of oxidation brought about a greater disruption of the $\mathrm{sp}^{2}$ domains in the 6-day synthesized GO. The period of oxidation however, did not significantly affect the $\mathrm{I}_{\mathrm{D}} / \mathrm{I}_{\mathrm{G}}$ ratio of both synthesized GO. The optical properties showed that the 6-day synthesized GO has a much higher band gap value of $3.0 \mathrm{eV}$ compared to $2.5 \mathrm{eV}$ for the 3-day synthesized GO.

The 6-day synthesized GO produced graphene sheets with increased defect, more oxidized carbon, and higher band gap value which might reduce effectiveness in their use for energy applications. The contrary is the case for 3-day synthesized GO which showed lower band gap value and improved properties.

\section{Acknowledgements}

Our Sincere appreciation goes to Dr. V. T. Albert of Asbury Graphite Mills, New Jersey, USA for the supply of the Natural Flake Graphite for this research, and Microscopy unit of the University Of Pretoria, South Africa for the characterization of some of our samples.

\section{References}

[1] Huang, N.M., Lim, H.N., Chia, C.H., Yarmo, M.A. and Muhamad, M.R. (2011) Simple Room-Temperature of High Yield Large Area Graphene Oxide. International Journal of Nanomedicine, 6, 3443-3448. https://doi.org/10.2147/IJN.S26812

[2] Eluyemi, M.S., Eleruja, M.A., Adedeji, A.V., Olofinjana, B., Fasakin, O., Akinwun- 
mi, O.O., Ilori, O.O., Famojuro, A.T., Ayinde, S.A. and Ajayi, E.O.B. (2016) Synthesis and Characterization of Graphene Oxide and Reduced Graphene Oxide Thin Films Deposited by Spray Pyrolysis. Graphene, 5, 143-154.

https://doi.org/10.4236/graphene.2016.53012

[3] Yong, Z., Qiaoliang, B., Lena, A.L.T., Yulin, Z. and Kian, P.L. (2009) Hydrothermal Dehydration for the "Green" Reduction of Exfoliated Graphene Oxide to Graphene and Demonstration of Tunable Optical Limiting Properties. Chemistry of Materials, 21, 2950-2956. https://doi.org/10.1021/cm9006603

[4] Vorrada, L., Krit, T., Passakorn, E., Wanchai, B. and Achanai, B. (2013) Preparation and Characterization of Reduced Graphene Oxide Sheets via Water-Based Exfoliation and Reduction Methods. Advances in Material Science and Engineering, 2013, Article ID: 923403.

[5] Ban, F.Y., Majid, S.R., Huang, N.M. and Lim, H.N. (2012) Graphene Oxide and Its Electrochemical Performance. International Journal of Electrochemical Science, 7, 4345-4351.

[6] Pei, S. and Cheng, H.M. (2011) The Reduction of Graphene Oxide. Carbon, 50, 3210-3228. https://doi.org/10.1016/j.carbon.2011.11.010

[7] Sabina, D., Roksana, M., Agnieszka, S., Tadeusz, P., Kotyczka-Moranska, M. and Maciej, S. (2016) Studies of Reduced Graphene Oxide and Graphite Oxide in the Aspect of Their Possible Applications in Gas Sensors. Sensors, 16, 1-16.

[8] Asha, R.P. and Bipin, N. (2013) Synthesis of Reduced Graphene Oxide Using Novel Exfoliation Techniques and Its Characterization. Journal of Nano and Electronic Physics, 5, 2032-2034.

[9] Lim, H.N., Huang, N.M., Lim, S.S., Harrison, I. and Chia, C.H. (2011) Fabrication and Charcterization of Graphene Hydrogel via Hydrothermal Approach as a Scaffold for Preliminary Study of Cell Growth. International Journal of Nanomedicine, 6, 1817-1823. https://doi.org/10.2147/IJN.S23392

[10] Paulchamy, B., Arthi, G. and Lignesh, B.D. (2015) A Simple Approach to Stepwise Synthesis of Graphene Oxide Nanomaterial. Nanomedcine and Nanotechnology, 6, 253-256.

[11] Choi, Y.-J., Kim, E., Han, J.W., Kim, J.-H. and Gurunathan, S. (2016) A Novel Biomolecule Mediated Reduction of Graphene Oxide: A Multifunctional Anti-Cancer Agent. Molecules, 21, 375-394.

https://doi.org/10.3390/molecules21030375

[12] Christelle, P.P.W., Chin, W.L., Kian, M.L. and Sharifah, B.A.H. (2015) Advanced Chemical Reduction of Reduced Graphene Oxide and Its Photocatalytic Activity in Degrading Reactive Black 5. Materials, 8, 7118-7128. https://doi.org/10.3390/ma8105363

[13] Hayes, W.I., Joseph, P., Mughal, M.Z. and Papakonstantinou, P. (2014) Production of Reduced Graphene Oxide via Hydrothermal Reduction in an Aqueous Sulphuric Acid Suspension and Its Electrochemical Behaviour. Journal of Solid State Electrochemistry, 19, 361-380.

[14] Choudhuri, S. (2012) Bulk Synthesis of Graphene Nanosheets. B.Tech Thesis, National Institute of Technology, Rourkela.

[15] Grzegorz, S., Jaroslaw, S., Joanna, J., Rafal, K., Mariusz, Z., Marcin, H., Piotr, P., Jakub, B., Ludwika, L. and Krzysztof, M.A. (2011) Graphene Oxide vs. Reduced Graphene Oxide as Saturable Absorbers for Er-Doped Passively Mode-Locked Fiber Laser. Optics Express, 20, 19463-19473.

[16] Swarnima, K., Shashank, M. and Shantanu, K.B. (2014) Aqueous Colloidal Stability 
of Graphene Oxide and Chemically Converted Graphene. Journal of Nanoparticles, 2014, Article ID: 640281.

[17] Cao, L., Sushant, S., Parambath, A., Chang, Y.K. and Ya-Ping, S. (2012) Linear and Nonlinear Optical Properties of Modified Graphene-Based Materials. Materials Research Society Bulletin, 37, 1283-1289. https://doi.org/10.1557/mrs.2012.178 